\title{
Acupoint catgut embedding regulates GABA- Glutamate of menopausal panic disorder patients: protocol for a vivo study
}

\section{Guizhen Chen}

Guangzhou University of Chinese Medicine

\section{Shuo Zhang}

Guangzhou University of Chinese Medicine

\section{Xue Wang}

Guangzhou University of Chinese Medicine https://orcid.org/0000-0002-8205-7240

\section{Xiaokang Xu}

Guangzhou University of Chinese Medicine

\section{Zhen Zeng}

Guangzhou University of Chinese Medicine

\section{Yunxiang Xu ( $\nabla$ xuyx1968@163.com)}

Guangzhou University of Chinese Medicine https://orcid.org/0000-0002-6928-3390

\section{Method Article}

Keywords: Acupoint catgut embedding, GABA-Glutamate, Menopausal, Panic disorder, Protocol

Posted Date: January 31st, 2019

DOI: https://doi.org/10.21203/rs.2.262/v1

License: (1) This work is licensed under a Creative Commons Attribution 4.0 International License. Read Full License 


\section{Abstract}

Background: Around $10 \%$ of general population suffer from panic attack (PD), and menopausal women are high-risk group of PD. PD severely effects patients' living quality and social function because of its sudden onset and strong sense of impending death. The aim of this study is to investigate the pathogenesis, physio-pathology characters and seek for valid therapeutic measures.

Methods: A parallel designed, exploratory randomized controlled trial with 7:3 allocation to intervention group (contains Group Implantation and Group Medication) versus control group. The included women of Group Implantation will receive a biweekly acupoint catgut embedding treatment during 12 consecutive weeks in the following predefined acupuncture points: BL23, SP6, RN4, BL18, ST36 and BL20. All acupuncturists are medical doctors educated in acupuncture. The included women of Group Medication will take $0.4 \mathrm{mg}$ Alprazolam tablet orally, 1 tablet a time, 3 times a day. There is a study period of 3 months and a follow-up period of 1 month for each group. The primary outcomes are measured by the following therapeutic indexes: frequency of panic attack during observation period ( 3 months) and during follow-up period (1 month), Panic Disorder Severity Score (PDSS), Panic Associated Symptoms Score (PASS) and HAMA Score. The secondary outcomes are measured by the following therapeutic indexes: SLC-90 Score, Chinese Menopause Rating Scale Score. And there are several Biomarker Indexes: fMRI and $1 \mathrm{H}-\mathrm{MRS}$ scans taken pretherapy and post-treatment, free amino acid and E2 of peripheral blood tested pretherapy and post-treatment. Moreover, there are also safety indexes: blood routine test, urine routine test, stool routine test, liver function (ALT), kidney function (serum creatinine) and electrocardiogram.

Discussion: In this study, we are meant to reveal neural activity and amino acid metabolism of PD patients, and the internal relationship with clinic curative effect by using fMRI and $1 \mathrm{H}-\mathrm{MRS}$. The results of this study may provide a novel treatment regimen for PD patients.

Trial registration: Chinese Clinical Trial Registry, ChiCTR-INR-16009724, registered in November 2016.

Keywords: Acupoint catgut embedding, GABA-Glutamate, Menopausal, Panic disorder, Protocol

\section{Background}

General menopausal panic disorder is also called Acute Anxiety Attack (AAA), which is one of the main patterns of manifestation of anxiety disorder. Around $10 \%$ of general populations suffer from panic attack (PD), and menopausal women are high-risk group of $P{ }^{[1]}$. The main features of PD are repeated outbreak of panic, tachypnea, dizziness, palpitation, tremor and sweating. Because of its sudden onset and strong sense of impending death, PD severely affects patients' living quality and social function. These patients run around to seek medical advice, repeatedly run all kinds of auxiliary examinations, which not only lead to waste of medical resources, but also bring heavy financial burden for families and society. 
Modern medicine towards menopausal panic disorder such as Hormone replacement therapy, no hormonal medication therapy, cognitive behavioral therapy and etc have many flaws like variety of side effects, high recurrence rate, drug dependence and so on ${ }^{[2]}$.

The therapy combining drugs and cognitive behavioral therapy (CBT) has become a hot research area of treating PD recently, which shows great clinical effects in short terms. However, there are many flaws in long terms. For example, objects are really easy to fall off after drug withdrawal. So its efficacy has not been proved yet.

Therefore, investigating the pathogenesis, physio-pathology characters and seeking for valid therapeutic measures are quite essential to menopausal patients, for both their body and mind health care.

\section{Clinical appliance}

Acupoint catgut embedding can alleviate symptoms of menopausal panic disorder and improve the life quality ${ }^{[3]}$. It is likely related to physiological functions of neural activity. However, researches of this treatment have not involved its effect mechanism and targets yet.

\section{Research necessity}

Acupoint catgut embedding can modulate amino acid closely related to emotion regulation of OVX rats $^{[3]}$. The drop of inhibitory GABA, which leads to imbalance of Glutamate-GABA, is known as the basic cause of $P D^{[4,5]}$. However, whether or not that PD patients' neural activity status and neural conductive mechanism care about the drop of inhibitory GABA still remains unreported. This study opens the door to verify pathogenesis of menopausal panic disorder and empolder effective control measures.

\section{Hypothesis}

The main aetiological agent of menopausal panic disorder is the metabolic disorder of amino acid and it causes the excitatory and inhibitory imbalance of brain function ${ }^{[6]}$. However, acupoint catgut embedding is able to correct the metabolic flaws of fear center and promote the recovery of fear network, therefore adjust neural activity.

\section{Methods}

\section{Study design}

This is a parallel designed, exploratory randomized controlled trial with 7:3 allocation to intervention group (contains Group Implantation and Group Medication) versus control group. The study is designed to investigate central nervous mechanism of menopausal panic disorder patients with treatment of acupoint catgut embedding, reveal neural activity and amino acid metabolism of PD patients, and internal relationship with clinic curative effect by the use of fMRI and 1H-MRS. The report follows the National Standards of P.R (GB/T 21709.10-2008) manipulations of acupuncture and moxibustion-Part 
10 Acupoint Catgut Embedding. The protocol adheres to the SPIRIT 2013 statement; further details are in the Additional file 1. The trial will be done in Bao'an TCM Hospital of Guangzhou University of Chinese Medicine.

\section{Recruitment}

There are 3 ways to recruit participants: 1 Recruit from clinic patients of 3 cooperative hospitals. We will gather each hospital's director of acupuncture department and gynecology department and call a seminar focusing on how to recruit participants. Then we will send assistants to each department to help screen participants, in the meanwhile put up a post about this study to attract potential candidates. 2 Recruit via broadcasting, TV and newspapers, etc. We will give a brief description about our expecting participants and provide free examinations and treatments to those who meet the standards in these advertisements. 3 Attract suitable participants by introducing some basic knowledge about menopausal physiology, menopausal pathology and its treatment in community service.

\section{Study subject}

All the subjects are menopausal syndrome specialist clinic patients from menopausal syndrome clinical research base of Guangdong Traditional Chinese Medicine and Affiliated Bao'an TCM Hospital of Guangzhou University of Chinese Medicine. And healthy female volunteers who are in normal childbearing age and from the same region act as the control group. Study subjects will receive oral and written information and must sign an informed consent form before enrolment. The inclusion and exclusion criteria are presented in Table 1.

\section{Baseline}

In a baseline questionnaire, information will be obtained about age, occupation (physical/non-physical), education level, last menstrual period, previous use of acupuncture, expectation towards acupoint catgut embedding. Moreover, PDSS, PASS, HAMA Score, SLC-90 Score must be completed at baseline (week 0). Thereafter, the study subjects will be randomized.

\section{Randomization}

Random numbers are generated by PEMS 3.1, and made into random cards. Then the cards are sealed in impellucidus envelopes, kept by designated personnel. There is a one-to-one correspondence between the registration of clinic patients and the serial number of the envelope which contains the random number. Based on the number in the envelope each patient gets, these 70 patients are grouped into Group Implantation and Group Medicine at random, and each group contains 35 samples. In the meanwhile, healthy female volunteers who are in normal child-bearing age are chosen as the control group.

\section{Blinding}

Since it is unachievable to apply blind method to experimental subjects of two groups above and the experimenters, this study propose to apply blind method to result measurers and data statisticians. 


\section{Intervention and setting}

The study will take place in Affiliated Bao'an TCM Hospital of Guangzhou University of Chinese Medicine. Patients of Group Implantation get one-time acupoint catgut embedding every 2 weeks, while patients of Group Medication take Alprazolam (Guangxi Southpearl Pharmaceutical Co., Ltd.) with size of $0.4 \mathrm{mg}$ tablet orally, 1 tablet a time, 3 times a day. There are 3 months study period and 1 month follow-up period for each group. Meanwhile, there is no intervention towards healthy female volunteers who are in normal child-bearing age in control group, except one index examination the day they join the study. A more detailed process flow can be seen in Fig. 1.

\section{Medical material information}

The needles will be sterile disposable No.8 injection needles size $0.8 \times 38$ TW LB (Zhejiang KangKang Medical-Devices Co., Ltd.). The core needles will be sterile disposable No.28 acupuncture needles size $0.35 \times 50 \mathrm{~mm}$ (Rui Qier) used after their pinpoints rubdown. The catgut will be absorbable surgical suture (medical catgut), size 3/0 (Shanghai Pudong Jinhuan Medical Products Co., Ltd.).

\section{Acupuncture points}

Main acupoint: BL23, SP6, RN4.

If it's a liver-kidney yin deficiency case, add BL18.

If it's a kidney yang deficiency case, add ST36 and BL20.

In each treatment, RN4 is a required acupoint, and the rest acupoints can be used in alternation of left/right side.

Location method: according to National Standards of P.R < Location of Acupoints > promulgated by the State Bureau of Technology Supervision. Specific acupoints locations are as followed. (Table 2)

\section{Acupoint Catgut Embedding}

Operating method (implantation with needles): according to National Standards of P.R (GB/T 21709.102008) manipulations of acupuncture and moxibustion-Part 10.

- Cut the $3 / 0$ catgut into $1.5 \mathrm{~cm}$ segment and soak them in disinfectant.

- During the treatment, patients take prone position to get back-shu point implantation and supine position to get RN4, SP6 and ST36 implantation. Based on location method above, disinfect routinely at acupoint and its skin around with Anerdian, take one self-made embedding needle, stick the core into needle tubing, and then pull out the core about $2 \mathrm{~cm}$, implant one $1.5 \mathrm{~cm}$ sterile catgut into the front of the tubing, and tighten or pinch the local skin around the acupoint with left thumb and forefinger, stick the needle into the acupoint rapidly with the right hand, and the lift and down the 
needle until acquire de qi. Push the core and withdraw the tubing slowly, and finish implanting the catgut deep down the acupoint. Hold pressure for a moment after withdraw the needle, check for exposure of catgut out of skin and bleeding, cover the wound with a piece of gauze or band-aid for 12 days.

- Direction, angle and depth of implantation: BL23, SP6, RN4 are required vertical puncture into the acupoint for about $0.8-1.0$ cun (approximately $20 \mathrm{~mm}-25 \mathrm{~mm}$ ); ST36 is required to vertical puncture into the acupoint for about 1.0-1.5 cun (approximately $25 \mathrm{~mm}-40 \mathrm{~mm}$ ); BL20 is required vertical puncture into the acupoint for about $0.5-0.8$ cun (approximately $13 \mathrm{~mm}-20 \mathrm{~mm}$ ); BL18 is required oblique puncture towards the spine into the acupoint for about $0.5-0.8$ cun (approximately $13 \mathrm{~mm}$ $20 \mathrm{~mm})$.

\section{fMRI examination procedure}

The MRI examination will be performed to show changes of brain's function directly and timely through the blood oxygen level imaging which can offer visualized proof. The parameters of the MRI (Siemens) protocol are as followed: high-resolution three-dimensional T1 weighting brain volume (Bravo) sequence scanning with repetition time (TR) of $8.16 \mathrm{~ms}$, echo time (TE) of $3.18 \mathrm{~ms}$, flip angle of $7^{\circ}$, field of view (FOV) of $256 \times 256 \mathrm{~mm}^{2}$, voxel size of $1 \times 1 \times 1 \mathrm{~mm}^{3}$, number of slices of 180 and slice thickness of 1.0$\mathrm{mm}$. The subjects will be asked to keep quiet and their eyes closed. Meanwhile, they need to empty their minds but avoid sleep. The researcher will check whether the participants are awake during the scanning procedure.

\section{Outcome}

Outcomes will be evaluating using multiple indexes. The primary outcomes are measured by the following therapeutic indexes: frequency of panic attack during observation period (3 months) and during follow-up period (1 month), Panic Disorder Severity Score (PDSS), Panic Associated Symptoms Score (PASS), and HAMA Score. The secondary outcomes are measured by the following therapeutic indexes: SLC-90 Score, Chinese Menopause Rating Scale Score. And there are two Biomarker Indexes: $\mathrm{fMRI}$ and $1 \mathrm{H}-\mathrm{MRS}$ scans taken pretherapy and post-treatment, free amino acid and E2 of peripheral blood tested pretherapy and post-treatment.

fMRI will focus on the activity of Amygdaloid necleus and the connections between fear center and fear network to reflect the changes of brain's function of menopausal panic disorder patients before and after treatment. 1H-MRS will test Glutamate-GABA level of these brain tissues to explore the relations between neural activity and amino acid metabolism.

All study subjects will be measured by all those indexes except PASS. Frequency of panic attack, Panic Disorder Severity Score (PDSS), HAMA Score, SLC-90 Score, and Chinese Menopause Rating Scale Score will be measured every 4 weeks, and those two Biomarker Indexes will be tested two times: in week 0 and week 12 . 


\section{Power calculations}

Based on preliminary experiments, it is obtained that after treatment the kuppermann index (MI) of Group Acupoint Catgut Embedding drops 15.90 \pm 2.71 , while Group Medication drops $13.84 \pm 1.87$, and the population standard deviation $S=2.12, \delta=2.06$; given $\alpha=0.05$ bilateral, $\beta=0.1$, power of test $1-\beta=0.9$. Based on formula ${ }^{[7]}, \mathrm{n}=2 \mathrm{x}[(\mathrm{ta}+\mathrm{t} 2 \beta) \mathrm{s} / \delta] \times[(\mathrm{ta}+\mathrm{t} 2 \beta) \mathrm{s} / \delta]$, calculating with trial and error method, and use table look-up: ta, $\infty=1.96, t 2 \beta, \infty=1.282$, then we obtain $n=23.66 \approx 24$, then according to t table: t0.05 $(24 \times 2$ $2)=2.0129, t 0.2(24 \times 2-2)=1.3002$, then substitute it into the former formula, we obtain $n=23.25 \approx 24$, the sample size has plateaued, and the attempt stop. Which means each group requires 24 samples, and two groups require 48 samples in total $(n=48)$, with the consideration of maximum lost rate of follow up is $20 \%$, we conclude that the total sample size is 60 . According to HAMA (which contains 14items), the sample size is $14 \times 5=70$. Take two parts above into consideration, the sample size is determined as 70 .

\section{Statistical analysis}

Image data processing: using methods like independent component analysis (ICA) and function binding, applying professional softwares such as MRIcro for windows, Xjview 8.1, SPM 8 and Matlab 5.1.

Statistical analysis technique: applying statistical test to difference of prior-treatment and posttreatment, examining the curative effect difference within groups and among groups of all curative effect index. We adapt two-sided test for all statistical tests, it's considered that the tested differences are statistically significant if $P$ less than or equal to 0.05 , while if $P$ greater than or equal to 0.05 , then the tested differences are not statistically significant. Details are as follows:

Measurement data: adapt t-test to run comparison among groups, when it doesn't comply with the normal distribution, we change into Wilcoxon rank-sum test. We run paired t-test towards difference of prior-treatment and posttreatment, and we change into Wilcoxon rank-sum test while it doesn't comply with the normal distribution.

Enumeration data: we use Chi-square test, calibration Chi-square test, and Fisher exact method to run comparison among groups.

Ranked data: we use Wilcoxon rank-sum test to run comparison among groups and signed rank sum test to run comparison within groups.

\section{Adverse events}

In the research, some adverse reactions may occur. We will monitor every patient with adverse reaction. If patient feel any discomfort during interviews, please contact and consult your research physician in time.

There are a few common responses after the acupoint catgut embedding:

- Local reaction: Mostly some sterile inflammations with inflamed hot pain occur within 5 days. Some cases may be more sever, like a small amount of milky-white exudation at the implantation spot 
caused by fat liquefaction because of catgut stimulation. Circumstances above should not require any special treatment.

- General reaction: Some patients may experience temperature rising after the treatment 4 to 24 hours, which mostly around $38^{\circ} \mathrm{C}$. It will fade away in 2 to 4 days by itself. Usually hemogram of each patient may rise in different levels, and it will come back to normal in 3 to 5 days.

There are a few adverse responses after the acupoint catgut embedding:

1. Pain: Apply hot compress if there is pain at implantation acupoint.

2. Secondary infection: Lack of strict asepsis and well protection of the wound could cause secondary infection, which mostly occurs some inflammatory symptoms like local swelling, progressive pain, and fever 3 to 4 days after the implantation. Apply hot compress and anti-infective treatment would help.

3. Nerve injury: It usually caused by incorrect operation or excessive stimulation or carelessness. It can be avoided with careful operation.

4. Hemorrhage: It usually caused by puncture on the vessels or excessive stimulation, in which case pressure dressing on the puncture point would stop the bleeding.

5. Catgut allergy: Local itching or swelling or fever can be remitted by antianaphylaxis treatment.

If any of above adverse responses occurs, participants should contact their research physicians in time, and they will offer corresponding treatment and advice. If there are any notable findings that may affect the continuation of this research, subjects or their legal representatives will be notified.

Patients need to inform their family or close friends that they are participating in a clinical research, and they can pay attention to adverse responses presented above. If they have any questions about this study, subjects can offer the contact information of their research physicians.

There are some risks of Alprazolam: Some patients will use alprazolam in the research (medication group). Alprazolam is one of Benzodiazepines, and its principle effects are antianxiety and antidepressant. Common adverse reactions are as followed:

1. a) Somnolence, vertigo, and weakness etc, large dose may cause dystaxia, tremor, uroschesis or jaundice.

2. b) Alprazolam is addictive. Drug withdrawal may cause withdrawal symptoms after using for a long period, showing as emotional or melancholy.

3. c) A few patients may suffer dry mouth, inattention, hyperhidrosis, palpitation, constipation or diarrhea, blurred vision, hypotension.

If participants have any questions about having Alprazolam, they could consult their research physician.

\section{Quality control}


All the investigators have been trained well to follow the trial's procedure and acupuncturists are instructed to give the acupuncture treatment merely. Any other treatment or counseling is forbidden. To ensure the quality of the study, the clinical monitors will arrange specialized staffs to check the process of the trial and document the details of medicine use. Additionally, monitors nominated by the principal investigator will check the accuracy and validity of the original data which should be uploaded in time and accompanied with text summaries. Regular meetings will be held to handle with the difficulties and problems emerging during the study.

\section{Discussion}

This study is a parallel designed and exploratory randomized controlled trial with 7:3 allocation to intervention group (contains Group Implantation and Group Medication) versus control group which is sponsored and financially supported by National Natural Science Foundation of China. The purpose of this study is to reveal neural activity and amino acid metabolism of PD patients, and internal relationship with clinic curative effect by using fMRI and 1H-MRS.

Panic disorder is also known as acute anxiety attack (AAA), which is one of the main patterns of manifestation of anxiety disorder. Around $10 \%$ of general populations suffer from panic attack (PD), and menopausal women are high-risk group of PD. The main features of PD are repeated outbreak of panic, tachypnea, dizziness, palpitation, tremor and sweating. It falls under the category of 'Kong', which belongs to five emotions in Traditional Chinese Medicine. Because of its sudden onset and strong sense of impending death, it severely affects patients' living quality and social function. There are many studies show different mechanisms in this disease, our laboratory runs a variety of researches aiming at GAMA mechanism in long terms. And in this mechanism, free amino acid is an iconic index. Besides, study shows that there are changes in $\mathrm{fMRI}$ images of menopausal panic disorder.

Based on structural data from human and animal research, brain-imaging studies of the neural correlates of emotional processing identified a network of structures which constitutes the neural circuitry for emotions, including the amygdala, cingulate, insula and prefrontal cortex (PFC). They interact to distinguish the emotional significances of the stimuli and to generate and regulate affective states ${ }^{[8,9]}$. Amygdaloid nucleus is described as the "fear center" of the brain or the core of "fear network"[10]. Studies have shown that PD patients have an excessive activity of amygdaloid nucleus and HypothalamusPituitary-Adrenal axis (H-P-A axis). With the improvement of the disease, the activity of amygdaloid nucleus tends to be normal. Prefrontal cortex participates in emotional processing. Anterior cingulate cortex is closely related to mood and self-control ${ }^{[11]}$. As the connecting hub, insular lobe connects with the anterior cingulate cortex, amygdaloid necleus, prefrontal cortex and other tissues which are also form the circuit. Insula hyperreactivity is associated with processing negative emotional information ${ }^{[12-14]}$, threat-relevant cues and symptom severity ${ }^{[15]}$. Structural phase study found abnormal volume of insula gray matter, anterior cingulate cortex and amygdaloid necleus occur in PD patients. Functional phase 
studies revealed that abnormal activities of amygdaloid nucleus, anterior cingulate cortex and prefrontal cortex lead to cognitive loss and predictive anxiety ${ }^{[16,17]}$, suggesting the close connection between occurrence and development of panic disorder and neuro-anatomic structures.

After successful treatment with cognitive behavioural therapy (CBT), previous studies discovered changes of brain activation in cortico-limbic structures, particularly an inhibitory functional coupling between the ACC and the amygdala concerning the response to treatment ${ }^{[18]}$. Studies have shown that precuneus and amygdaloid nucleus in patients with panic disorder are related to anterior cingulate, and are negatively correlated with $\gamma^{-G A B A}$ levels in the anterior cingulated ${ }^{[19]}$. Neurological imaging studies have shown that Glutamate and GABA are the most important amino acid neurotransmitters, maintaining brain's excitement and inhibition and mediating the conduction of more than $90 \%$ of brain's nerve. Accordingly, we hypothesize that the metabolic disorder of amino acid, more accurately the imbalance of Glutamate-GABA caused by GABA's content decreased may be the main aetiological agent of menopausal panic disorder, causing the excitatory and inhibitory imbalance of brain function ${ }^{[20]}$.

This study aims at investigating the pathogenesis and physio-pathology characters by revealing neural activity and amino acid metabolism of PD patients, and internal relationship with clinic curative effect by using fMRI and 1H-MRS, seeking for valid therapeutic measures as well.

Modern medicine towards menopausal panic disorder have bunch of disadvantages, for example, hormone replacement therapy could increase the risk of higher morbidity of endometrial cancer, breast cancer and colorectal cancer. Nonhormonal medication therapy (benzodiazepines, tricyclics antidepressant, monoamine oxdase inhibitor, etc) may lead to nausea and vomiting, dizziness and headache, weakness and tremor, change of weight. The therapy that combines drugs and cognitive behavioral therapy (CBT) has become a hot research area of treating PD recently, which shows great clinical effects in short terms. However, there are many flaws in long terms, for instance, objects are really easy to fall off after drug withdrawal. So its efficacy has not been proved yet. Therefore, these existing treatments either have little efficacy or have many side effects.

'Keep it for a long time to cure chronic diseases' is the theoretical guidance of acupoint catgut embedding therapy. This therapy developed by using modern technology based on traditional acupuncture technique .It takes advantage of the stimulation of acupoint via physicochemical change of the catgut in human body. It is a comprehensive regulative process. Studies show that catgut embedding therapy has received great efficacy in menopausal panic disorder.

An advantage of our study is that early studies show significant efficacy without side effects. Many previous studies are lack of objective index to reveal and measure the functions of the treatment, but our study will include fMRI and 1H-MRS technology as strong evidence.

Unfortunately, at present the syndrome differentiations of TCM are not clear yet, and diagnostic criteria and curative effect evaluation have no unified standard, and related researches are limited in short treating period. All these factors restrict our study. 
In conclusion, the results of this trial are expected to reveal the pathogenesis and physio-pathology characters of PD, seek for valid therapeutic measures such as catgut embedding as we perform.

With the imaging of fMRI and 1H-MRS technology, we expect to show neural activity and amino acid metabolism of PD patients, and internal relationship with clinic curative effect. We believe the outcome will exert a positive effect on acupoint catgut embedding treatment for menopausal panic disorder.

\section{Abbreviations}

HAMA: Hamilton Anxiety Scale

SLC-90: Symptom Checklist 90

fMRI: Functional magnetic resonance imaging

1H-MRS: Proton magnetic resonance spectroscopy

OVX: Ovariectomized

GABA: Gamma-aminobutyric acid

TCM: Traditional Chinese Medicine

\section{Declarations}

\section{Trial status}

Version number of the protocol: 15010000002006495

Recruitment for the trial started in December 2017 and is expected to end in December 2021.

\section{Ethics approval and consent to participate}

The protocol was approved by Ethics Committee of Affiliated Bao'an TCM Hospital of Guangzhou University of Chinese Medicine in February, 2014. Written informed consent will be obtained from all study patients before study enrollment. After each qualified patient consents to participate in, the enrollment and data collection will be started. And informed consent was formulated.

\section{Consent for publication}

Not applicable

\section{Availability of date and material}

Not applicable 


\section{Competing interests}

The authors declare that they have no competing interests. The authors declare no financial or vested interest in the company providing the medicines or instruments.

\section{Funding}

The authors thank the National Natural Science Foundation of China (grant No: 81574064; 81473755), the Scientific Research Program of Administration of Traditional Chinese Medicine of Guangdong Province (grant No: 20181237), the Xinhuo Planning Project of Guangzhou University of Chinese Medicine (grant No: XH20150106), and the Shenzhen Science and Technology Planning Project (grant No: JCYJ201703063000328).

\section{Authors' contributions}

GZC has led, coordinated and wrote the manuscript, designed the study, and developed the methodology. SZ and XW have supported and contributed in the manuscript writing, and tables, and images design. YXX contributed to the development and refinement of the study protocol. XKX and ZZ made substantial contributions to the conception and design of the study and subsequent protocol revisions. All authors read and approved the final manuscript.

\section{Acknowledgements}

Not applicable

\section{Authors' information}

Guizhen Chen, Postdoctoral Fellow, Researcher, Master Tutor, Affiliated Bao'an TCM Hospital, Guangzhou University of Chinese Medicine, Shenzhen, 518101, China.

Shuo Zhang, Bachelor, Master Candidate, Clinical Medical College of Acupuncture and Rehabilitation, Guangzhou University of Chinese Medicine, Guangzhou, 510405, P. R. China.

Xue Wang, Bachelor, Master Candidate, Clinical Medical College of Acupuncture and Rehabilitation, Guangzhou University of Chinese Medicine, Guangzhou, 510405, P. R. China.

Xiaokang Xu, Bachelor, Master Candidate, Clinical Medical College of Acupuncture and Rehabilitation, Guangzhou University of Chinese Medicine, Guangzhou, 510405, P. R. China.

Zhen Zeng, Bachelor, Master Candidate, Clinical Medical College of Acupuncture and Rehabilitation, Guangzhou University of Chinese Medicine, Guangzhou, 510405, P. R. China.

Yunxiang Xu, Doctor of Medicine, Professor, Doctoral Supervisor, Clinical Medical College of Acupuncture and Rehabilitation, Guangzhou University of Chinese Medicine, Guangzhou, 510405, P. R. China. 


\section{References}

1. Kessler RC, Chiu WT, Jin R, Ruscio AM, Shear K, Walters EE. The epidemiology of panic attacks, panic disorder, and agoraphobia in the National Comorbidity Survey Replication. Arch Gen Psychiatry. 2006;63(4):415-24.

2. Xin W, Xinming L. Progress the in etiology and treatment of panic disorder. Chinese Journal of Clinical Pharmacology and Therapeutics. 2008;13(1):25-30.

3. Zhang L, Wang Y, Xu Y, Lei H, Zhao Y, Li H, et al. Metabonomic analysis reveals efficient ameliorating effects of acupoint stimulations on the menopause-caused alterations in mammalian metabolism. Scientific reports. 2014;4:3641.

4. Stork O, Ji FY, Kaneko K, Stork S, Yoshinobu Y, Moriya T, et al. Postnatal development of a GABA deficit and disturbance of neural functions in mice lacking GAD65. Brain Res. 2000;865(1):45-58.

5. Goddard AW, Mason GF, Appel M, Rothman DL, Gueorguieva R, Behar KL, et al. Impaired GABA neuronal response to acute benzodiazepine administration in panic disorder. Am J Psychiatry. 2004;161(12):2186-93.

6. Qing C, Dajun Y, Changxu S. The effect of estrogen on the hypothalamus. Chongqing Medicine. 2003;32(1):110-2.

7. Xiaobin Z, Jian Z, Dongfeng Z, Qiuzhen W. Sample Size Estimation in Medical Research. Acta Academiae Medicinae Qingdao Universitatis. 1998(2):155-6.

8. Phillips ML, Drevets WC, Rauch SL, Lane R. Neurobiology of emotion perception I: The neural basis of normal emotion perception. Biol Psychiatry. 2003;54(5):504-14.

9. Phillips ML, Drevets WC, Rauch SL, Lane R. Neurobiology of emotion perception II: Implications for major psychiatric disorders. Biol Psychiatry. 2003;54(5):515-28.

10. Feldker K, Heitmann CY, Neumeister P, Tupak SV, Schrammen E, Moeck R, et al. Transdiagnostic brain responses to disorder-related threat across four psychiatric disorders. Psychol Med. 2017;47(4):73043.

11. Vallesi A. Connectivity between ventromedial prefrontal cortex and posterior superior temporal sulcus. Cognitive neuroscience. 2016;7(1-4):24-5.

12. Shah SG, Klumpp H, Angstadt M, Nathan PJ, Phan KL. Amygdala and insula response to emotional images in patients with generalized social anxiety disorder. J Psychiatry Neurosci. 2009;34(4):296302.

13. Ball TM, Sullivan S, Flagan T, Hitchcock CA, Simmons A, Paulus MP, et al. Selective effects of social anxiety, anxiety sensitivity, and negative affectivity on the neural bases of emotional face processing. Neuroimage. 2012;59(2):1879-87.

14. Klumpp $H$, Angstadt $M$, Phan KL. Insula reactivity and connectivity to anterior cingulate cortex when processing threat in generalized social anxiety disorder. Biological Psychology. 2012;89(1):273-6.

15. Schmidt S, Mohr A, Miltner WH, Straube T. Task-dependent neural correlates of the processing of verbal threat-related stimuli in social phobia. Biol Psychol. 2010;84(2):304-12. 
16. Terasawa $Y$, Shibata M, Moriguchi $Y$, Umeda S. Anterior insular cortex mediates bodily sensibility and social anxiety. Soc Cogn Affect Neurosci. 2013;8(3):259-66.

17. Li Z, Wei L, Xinyi C, Chunbo L. Progress in imaging of leaf brain of anxiety disorder. Journal of International Psychiatry. 2015;1:035.

18. Lueken U, Straube B, Konrad C, Wittchen HU, Strohle A, Wittmann A, et al. Neural substrates of treatment response to cognitive-behavioral therapy in panic disorder with agoraphobia. Am J Psychiatry. 2013;170(11):1345-55.

19. Shin Y-W, Dzemidzic M, Jo HJ, Long Z, Medlock C, Dydak U, et al. Increased resting-state functional connectivity between the anterior cingulate cortex and the precuneus in panic disorder:: Resting-state connectivity in panic disorder. Journal of affective disorders. 2013;150(3):1091-5.

20. Wierońska JM, Stachowicz K, Nowak G, Pilc A. The loss of glutamate-GABA harmony in anxiety disorders. In: Kalinin VV, editor. Anxiety disorders: IntechOpen; 2011. p. 135-58.

\section{Tables}

Table 1 Inclusion and exclusion criteria 
Inclusion criteria

4 Corresponding to diagnosis standards of PD.

4. Female, aged 41 to 60, at least junior middle school literacy.

[ $\mathrm{HAMA} \geq 14$.

․ Patients who consent to sign informed consent form.

Exclusion criteria

૫ Combined with other mental disorders, such as phobia and melancholia, or panic disorder caused by somatoform disorder.

[ Combined with sever cardiac and hepatic and renal insufficiency, or other serious diseases of other systems like malignant tumor.

[ Panic disorder caused by physical diseases like epilepsy, heart attack, pheochromocytoma, hyperthyroidism, or spontaneous hypoglycemia, etc.

․ Patients who used antipsychotic drugs, antidepressive drugs, or sexual hormones within 2 weeks before included, or combined taking other therapy like cognitive behavioral therapy etc.

4 Patients who have contraindications to MR, like aneurismal clip, implantable neuro-stimulator, pacemaker, automatic implantable defibrillator, cochlear implantation or visual obstacles.

․ Female during pregnancy or lactation.

4 Patients who suffered from alcohol or drug abuse within 1 year or with a history of alcohol or drug dependency.

Table 2 Acupoints location. 
Point Location

BL23 1.5 cun lateral to the depression below the spinous process of the 9th vertebra lumbalis

SP6 Posterior to the mesial border of the tibia, and 3 cun above the tip of the medial malleolus

RN4 On the anterior midline,3 cun below the umbilicus

BL18 1.5 cun lateral to the depression below the spinous process of the 9th thoracic vertebra

ST36 3 cun directly below Dubi, and one finger-breadth lateral to the anterior border of the tibia

BL20 1.5 cun lateral to the depression below the spinous process of the 2 th thoracic vertebra

Cun $=$ an acupuncture measurement unit, 1 cun corresponds to the width of the study subject's thumb.

\section{Figures}




\begin{tabular}{|c|c|c|c|c|c|c|c|c|}
\hline \multirow{3}{*}{\multicolumn{2}{|c|}{ TIMEPOINT(week) }} & \multicolumn{7}{|c|}{ STUDY PERIOD } \\
\hline & & \multirow{2}{*}{$\begin{array}{c}\text { Enrollment } \\
-1\end{array}$} & \multirow{2}{*}{$\begin{array}{c}\text { Allocation } \\
0\end{array}$} & \multicolumn{4}{|c|}{ Post-allocation } & \multirow{2}{*}{$\begin{array}{c}\text { Follow-up } \\
13-16\end{array}$} \\
\hline & & & & $\mathbf{0}$ & 4 & 8 & 12 & \\
\hline \multicolumn{9}{|c|}{ ENOLLMENT } \\
\hline & Inclusion and Exclusion Criteria & $\mathrm{X}$ & & & & & & \\
\hline & Informed consent & $\mathrm{X}$ & & & & & & \\
\hline & General Conditions & $\mathrm{X}$ & & $\mathrm{X}$ & $\mathrm{x}$ & $\mathrm{X}$ & $\mathrm{X}$ & \\
\hline & PMH(Past Medical History) & $\mathrm{X}$ & & & & & & \\
\hline & Signs and Symptoms & $\mathrm{X}$ & & $\mathrm{X}$ & $\mathrm{X}$ & $\mathrm{X}$ & $\mathrm{X}$ & \\
\hline Tongue $\mathbf{M}$ & $\begin{array}{l}\text { Physical Examination: } \\
\text { estation, Pulse Manifestation, Weight, Vital Signs }\end{array}$ & $\mathrm{X}$ & & & & & $\mathrm{X}$ & \\
\hline Electrocardi & $\begin{array}{l}\text { Safety Examination: } \\
\text { am, Liver Function, Kidney Function, Blood Lipid }\end{array}$ & $\mathrm{X}$ & & & & & $\mathrm{X}$ & \\
\hline & ALLOCATION & & $\mathrm{x}$ & & & & & \\
\hline \multicolumn{9}{|c|}{ INTERVENTIONS } \\
\hline \multicolumn{9}{|c|}{ Acupoint Catgut Embedding } \\
\hline \multicolumn{9}{|c|}{ Drugs } \\
\hline \multicolumn{9}{|c|}{ OUTCOMES } \\
\hline \multirow{2}{*}{$\begin{array}{l}\text { Primary } \\
\text { Indexes: }\end{array}$} & Frequency of Panic Attack, PDSS, HAMA Score & $\mathrm{X}$ & & $\mathrm{X}$ & $\mathrm{X}$ & $\mathrm{X}$ & $\mathrm{X}$ & $\mathrm{X}$ \\
\hline & PASS & $\mathrm{X}$ & & & & & & \\
\hline \multicolumn{2}{|c|}{$\begin{array}{c}\text { Secondary Indexes: } \\
\text { SLC-90 Score, Chinese Menopause Rating Scale Score }\end{array}$} & $\mathrm{X}$ & & $\mathrm{X}$ & $\mathrm{X}$ & $\mathrm{X}$ & $\mathrm{X}$ & $\mathrm{X}$ \\
\hline fMRI and & $\begin{array}{l}\text { Biomarker Indexes: } \\
\text { MRS Scans, Free Amino Acid of Peripheral Blood }\end{array}$ & & & $\mathrm{x}$ & & & $\mathrm{x}$ & \\
\hline Collection & blood sample, urine sample and fecal sample & & & $\mathrm{X}$ & & & $\mathrm{X}$ & \\
\hline \multicolumn{9}{|c|}{ ASSESSMENTS } \\
\hline & Clinical Efficacy & & & $\mathrm{X}$ & & & $\mathrm{x}$ & \\
\hline & Compliance Assessment & & & $\mathrm{X}$ & $\mathrm{X}$ & $\mathrm{X}$ & $\mathrm{X}$ & \\
\hline & Adverse Events & & & $\mathrm{X}$ & $\mathrm{X}$ & $\mathrm{X}$ & $\mathrm{X}$ & \\
\hline & Safety Evaluation & & & $\mathrm{X}$ & & & $\mathrm{X}$ & \\
\hline
\end{tabular}

\section{Figure 1}

SPIRIT figure: schedule of enrollment, interventions and assessments.

\section{Supplementary Files}

This is a list of supplementary files associated with this preprint. Click to download. 
- supplement1.doc

Page 18/18 